\section{Systematische Leseförderung zur Verbesserung des flüssigen und sinnentnehmenden Lesens im Unterricht der Primarstufe}

\author{
Simone van Zadelhoff \\ Technische Universität Dortmund
}

In einem Dissertationsvorhaben, das im Lehrgebiet Rehabilitation und Pädagogik bei Lernbehinderungen an der Fakultät Rehabilitationswissenschaften der TU Dortmund durchgeführt wird, sollen zwei Lehr- und Lernarrangements zur Förderung des flüssigen und sinnentnehmenden Lesens entwickelt und in ersten Praxisversuchen vergleichend beurteilt werden. Das Projekt wird im Rahmen des Forschungs- und Nachwuchskollegs FUNKEN gefördert, das sich inhaltlich und methodisch an der Fachdidaktischen Entwicklungsforschung orientiert und die Entwicklung und iterative Erprobung und Verbesserung von Lehr-Lernarrangements für die Unterrichtspraxis anzielt (Prediger u.a. 2012).

\section{Theoretischer Hintergrund}

Das Lesen ist eine Fähigkeit, die wir mit dem Eintritt in das Schulleben erlernen und im weiteren Verlauf ausdifferenzieren und perfektionieren. In der heutigen Informationsgesellschaft stellt es das zentrale Medium der Wissensaneignung und des Lernens dar (vgl. Rosebrock/Nix 2011) und hat Auswirkungen auf unsere persönliche Weiterentwicklung und Teilhabe am gesellschaftlichen Leben. Die Vergleichsuntersuchungen DESI, IGLU und PISA haben jedoch gezeigt, dass es im deutschen Schulsystem eine recht große Gruppe Jugendlicher gibt, deren Leseleistungen unterdurchschnittlich entwickelt sind und denen somit schulisches und berufliches Versagen droht. Aus diesem Grund sind in der Lesedidaktik die Diskussionen und Forderungen nach neuen und erweiterten Konzepten von Leseförderungen laut geworden, die eine Fokussierung basaler Lesekompetenzen fordern. Besonders dem sinnentnehmenden und flüssigen Lesen kommt große Bedeutung beim Verstehen von Textinhalten zu, und es ergibt sich didaktisch die Notwendigkeit, schwachen Leseleistungen vorzubeugen oder gar entgegenzuwirken.

\section{Forschungsfragen und Zielsetzung}

Das Projekt hat die Zielsetzung, Lehr-Lernarrangements zur Förderung des sinnentnehmenden und flüssigen Lesens zu entwickeln und durch eine iterative Vorgehensweise optimal auf die schulische Praxis abzustimmen. Zwei Konzepte zur Förderung des flüssigen Lesens sollen in verschiedenen Sozialformen (Einzelförderung, Kooperative Lesepartnerschaften) des Unterrichts umgesetzt und Aussagen über die relative Wirksamkeit und Effektivität dieser Förderverfahren getroffen werden. Durch iterative Erprobung und Evaluation soll dasjenige Förderverfahren ermittelt werden, welches zu einer größeren Leistungssteigerung im Bereich des sinnentnehmenden und flüssigen Lesens führen kann bzw. das relativ zum zu erbringenden Aufwand vorrangig für die Schulpraxis empfohlen werden kann. Die Forschungspraxis wird durch folgende Fragen angeleitet:

1. Inwieweit kann eine systematische und diagnostisch fundierte Leseförderung zu einer Weiterentwicklung und Förderung der Leseleistungen von Lernenden beitragen?

2. Wie muss eine systematische und diagnostisch fundierte Leseförderung im kooperativen Lernsetting gestaltet sein, um eine Verbesserung der Leseleistungen von Lernenden zu erzielen?

3. Lassen sich unter beiden Bedingungen vergleichbare Effekte bei der Entwicklung der Leseleistungen erzielen?

\section{Forschungsdesign}

Die Stichprobe der Untersuchung setzt sich aus Lernenden der vierten Klasse mehrerer Grundschulen zusammen. An der Durchführung der Leseförderung im Einzelunterricht nehmen 23 Schülerinnen und Schüler, an der Umsetzung im kooperativen Lernen durch Lesepartnerschaften vier Grundschulklassen mit insgesamt 84 Lernenden teil. Um ein Lehr-Lernarrangement zu entwickeln, welches in der schulischen Praxis das sinnentnehmende und flüssige Lesen von Lernenden optimal fördern kann, wird ein Konzept zur Leseförderung in zwei unterschiedlichen Sozialformen des Unterrichts umgesetzt: im Einzelunterricht und in Kooperativen Lesepartnerschaften. 
Vor Beginn der Leseförderung im Einzelunterricht wurden die standardisierten Lesetests ELFE 1-6 und SLS 1-4 mit drei Grundschulklassen des vierten Schuljahres durchgeführt. Mittels der Ergebnisse dieser Tests, die die Lesegeschwindigkeit, die Lesegenauigkeit sowie das prosodische Lesen erheben, wurde die Stichprobe der ersten Intervention bestimmt. Es wurden 23 Lernende für die Förderung ausgewählt, deren Ergebnisse auf schwache bis sehr schwache Leseleistungen hindeuten. Nach der Auswahl der Stichprobe erhielt diese Gruppe über einen Zeitraum von 10 Wochen dreimal wöchentlich eine Leseförderung, die sich am individuellen Leseniveau der jeweiligen Lernenden orientierte. Durch eine individuelle und iterative Passung des Lesetextes mit ansteigendem Schwierigkeitsniveau sollten die Leseleistungen der Probanden verbessert werden. Nach Abschluss der Fördereinheit erfolgte eine erneute Durchführung der standardisierten Lesetests sowohl mit der Stichprobe der Untersuchung als auch mit den übrigen Lernenden des Klassenverbandes, um die Leseleistungen der Stichprobe mit den Leseleistungen der Kontrollgruppe vergleichen und in Beziehung setzen zu können. Um eine objektive Einschätzung des prosodischen Lesens zu erhalten, wird das laute Lesen der Lernenden anhand einer Skala zur Einschätzung des flüssigen Lautlesens erhoben und analysiert. Diese Daten und die begleitend erstellten Leseverlaufsdaten lassen Aussagen über die Wirksamkeit und die Effektivität des Förderverfahrens zu.

Für die zweite Umsetzung der Leseförderung wird das zuvor erprobte Konzept der Förderung im Einzelunterricht durch leichte Modifikationen in die Sozialform des Kooperativen Lernens durch Lesepartnerschaften eingepasst. Bei dieser Form der Leseförderung nehmen vier Grundschulklassen des vierten Schuljahres mit insgesamt 84 Lernenden teil. Wie bei der Umsetzung der Leseförderung im Einzelunterricht wurden auch hier zu Beginn der Förderung die standardisierten Lesetests ELFE 1-6 und SLS 1-4 durchgeführt. Die Zusammensetzung der Lesepaare erfolgte anhand der Ergebnisse dieser Tests, sodass sich jedes Lesepaar aus einem Mitglied mit starken (Begleiter) und einem mit schwachen (Leser) Leseleistungen zusammen- setzte. Die Lesepaare der Klassen führten die Leseförderung im Klassenverband unter Anleitung der Lehrperson über einen Zeitraum von sieben Wochen dreimal wöchentlich durch. Durch eine iterative Anpassung des Lesetextes an das individuelle Leseniveau des Lesers und durch einen schrittweisen Anstieg des Schwierigkeitsgrades wurde eine Verbesserung der Leseleistungen angestrebt. Nach Abschluss der Förderung erfolgte eine erneute Durchführung der standardisierten Lesetests. Diese Ergebnisse lassen Schlussfolgerungen über die Leistungssteigerung der Leser zu und erlauben Aussagen über einen möglichen Lernzuwachs der Begleiter. Zudem können durch die begleitend erstellten Leseverlaufsdaten und die objektive Einschätzung des prosodischen Lesens die Wirksamkeit und die Effektivität der Leseförderung im kooperativen Lernen bestimmt werden.

\section{Ausblick}

Die Durchführung der Leseförderung wurde im Spätsommer 2012 abgeschlossen, die Auswertung der quantitativen und qualitativen Daten wie auch die Analyse der individuellen Einzelfalldaten befinden sich in Arbeit. Welche der beiden Lernumgebungen erzielt eine größere Leistungssteigerung im Bereich des sinnentnehmenden und flüssigen Lesens? Wie und mit welchem Aufwand lassen sich die Förderverfahren in der unterrichtlichen Praxis einsetzen? Es ist zu hoffen, dass sich durch den Vergleich der Ergebnisse erste Aussagen über die relative Effektivität und Effizienz beider Förderverfahren in unterschiedlichen Sozialformen treffen lassen und dass darüber hinaus weitere Ansatzpunkte für eine Optimierung beider Lernumgebungen gewonnen und verschiedene Möglichkeiten effektiver Leseförderung entwickelt werden können.

Weitere Informationen und Literaturangaben können eingeholt werden bei simone.vanzadel hoff@tu-dortmund.de

Informationen über das Forschungs- und Nachwuchskolleg Funken finden sich unter http:// www.funken.tu-dortmund.de 


\section{Situation hörgeschädigter Jugendlicher in der Berufsausbildung auf dem ersten Arbeitsmarkt}

\author{
Markus Wild \\ Ludwig-Maximilians-Universität München
}

\section{Forschungshintergrund}

Das Forschungsprojekt „Situation hörgeschädigter Jugendlicher in der Berufsausbildung auf dem ersten Arbeitsmarkt" bildet das XV. Modul des am Lehrstuhl für Gehörlosen- und Schwerhörigenpädagogik der Ludwig-Maximilians-Universität München seit 1999 bestehenden Forschungsprogramms zur „Integration/Inklusion hörgeschädigter Kinder und Jugendlicher an allgemeinen Einrichtungen" (Leitung: Prof. Dr. habil. A. Leonhardt).

Die bisherigen Module untersuchten die Situation von Kindern und Jugendlichen mit Hörschädigungen im Kindergarten- und Schulalter (Schmitt 2003; Lindner 2007; Born 2009). Mit dem aktuellen Forschungsprojekt wird das Forschungsprogramm auf den Bereich der beruflichen Bildung ausgeweitet.

Weitere Forschungen im Kontext von Menschen mit Hörschädigungen im Arbeitsleben legen den Fokus auf gehörlose Arbeitnehmer bzw. sind älteren Datums (vgl. Landschaftsverband Rheinland 1993; Bungard/Kupke 1995; Musseleck/Musseleck 2000; Becker/Gelhardt 2003; Audeoud/Lienhard 2006; Sachsenhauser 2012). Die steigende Zahl der frühzeitig implantierten Cl-Träger findet darin noch keine Berücksichtigung, da diese erst jetzt das Ausbildungsalter erreichen.

\section{Forschungsziel und Forschungsfragen}

In der modernen Gesellschaft hat Arbeit nicht nur den Zweck der materiellen Existenzsicherung, sondern ihr kommen auch psychosoziale Funktionen zu (vgl. Semmer/Udris 2007). Für Menschen mit einer Hörschädigung ist wegen Letzterem ein Arbeitsverhältnis von besonderer Bedeutung (vgl. Menzel 2005). Neben dem angestrebten Beruf sind weitere Gründe denkbar, weshalb sich immer mehr Jugendliche mit Hörschädigung für eine Ausbildung auf dem ersten Arbeitsmarkt und damit gegen eine Ausbildung im Berufsbildungswerk (BBW) für Hörgeschädigte entscheiden. Expertengespräche zum Forschungsprojekt ergaben jedoch, dass der Besuch der allgemeinen Berufsschule dabei eine Hürde darstellen kann.

Ziel des Forschungsvorhabens ist es, erstmalig Informationen über die Situation peripher hörgeschädigter Jugendlicher in der Berufsausbildung im Ausbildungsbetrieb sowie an der allgemeinen Berufsschule zu erheben. Dabei soll festgestellt werden, wie die Kompensation der Hörschädigung und die Kommunikation in beiden Bereichen gelingt. Außerdem sollen Faktoren aus der Sicht der Jugendlichen erörtert werden, die die Integration in der Ausbildung bedingen können. Dabei werden die leistungsmotivationale, die soziale und die emotionale Dimension der Integration (vgl. Haeberlin u.a. 1989) berücksichtigt.

Aus der Zielsetzung der Forschungsarbeit ergibt sich folgende Fragestellung, die in drei Teilfragen aufgespaltet wird:

Wie gestaltet sich die Integration hörgeschädigter Jugendlicher in der Berufsausbildung auf dem ersten Arbeitsmarkt?

Wie ist die Situation hörgeschädigter Jugendlicher in der allgemeinen Berufsschule?

- Wie ist die Situation hörgeschädigter Jugendlicher im ausbildenden Betrieb?

Welche Faktoren bedingen die Integration?

\section{Forschungsdesign}

Um das Forschungsziel zu verfolgen, wurde ein qualitatives Forschungsdesign gewählt. Als Erhebungsinstrument für problemzentrierte Interviews wurde ein teilstandardisierter Interviewleitfaden entwickelt, der folgende Bereiche abfragte:

- schulische Laufbahn

- Ausbildungsverhältnis 
- Kommunikation und Kompensation der Hörschädigung in Berufsschule und Betrieb

- soziale, emotionale und leistungsmotivationale Situation in Berufsschule und Betrieb

- Mehraufwand und Nachteilsausgleich in Berufsschule und Betrieb

- Freizeitverhalten

Die offen formulierten Fragen des Leitfadens deckten die zu untersuchenden Bereiche ab und ermöglichten neue Aspekte. Die Erfassung der soziografischen Angaben erfolgte mit einem Kurzfragebogen.

Teilnehmer der Untersuchung waren Jugendliche mit peripherer Hörschädigung, die sich im Ausbildungsjahr 2011/12 im 1. bis 3. Lehrjahr einer Berufsausbildung in Bayern und Baden-Württemberg befanden. Für die Auswahl der Teilnehmer galten folgende Kriterien:

- ein Ausbildungsvertrag mit einem Betrieb des ersten Arbeitsmarktes

- der Besuch einer allgemeinen Berufsschule

- die Betreuung durch einen Fachdienst für Hörgeschädigte (Baden-Württemberg: Wohnortnahe Rehabilitation bzw. Sonderpädagogischer Dienst; Bayern: Unterstützte betriebliche Ausbildung bzw. Mobiler Sonderpädagogischer Dienst berufliche Schulen).

Über diese Fachdienste wurden 72 Auszubildende angeschrieben. Davon erklärten sich 23 aus Bayern und Baden-Württemberg zu einem Interview bereit. Vor der Erhebung wurde mit zwei Auszubildenden ein Pretest des Kurzfragebogens und des Interviewleitfadens durchgeführt. Da eine potenzielle Probandin im vorgegebenen Zeitraum nicht alle Kriterien erfüllte, konnten schließlich 20 Jugendliche im Alter von 16 bis 24 Jahren interviewt werden. Diese waren leichtgradig hörgeschädigt bis gehörlos. Zehn Jugendliche waren $\mathrm{Cl}$-Träger. Der überwiegende Teil wurde bereits integrativ beschult, Wechsel der Schulart waren jedoch nicht selten. Die Schulabschlüsse der Jugendlichen reichten vom Hauptschulabschluss bis zum Abitur, wobei die meisten die Mittlere Reife vorweisen konnten. Die gewählten Berufsbilder waren breit gefächert.
Alle Interviews wurden lautsprachlich geführt, digital aufgezeichnet und wortgetreu transkribiert. Die Auswertung erfolgt auf der Basis der qualitativen Inhaltsanalyse (vgl. Mayring 2010) unter Nutzung des Programms MAXqda2.

\section{Aktueller Stand der Erhebung}

Bei der Entscheidung für den gewählten Beruf und somit für die Ausbildung standen oft persönliche Interessen, Wohnortnähe des Betriebes und Sicherheit des Arbeitsplatzes im Vordergrund. Die Kontakte zu Ausbildern und Kollegen im Betrieb (soziale Situation) beschreiben die Jugendlichen teils sehr unterschiedlich. Viele empfinden ihre Kontakte zu den Kollegen und Vorgesetzten als „ganz normal“ und fühlen sich „voll dabei“. Andere nehmen ihre Kontakte als eher begrenzt wahr.

Auch bezüglich der Akzeptanz in der Berufsschule gehen die Meinungen auseinander. Manche bezeichnen ihre Mitschüler als „normale Freunde“, mit denen sie auch in ihrer Freizeit Kontakte pflegen. Andere beklagen, dass sie sich vor allem in informellen Situationen wie den Pausen ausgegrenzt fühlen.

Das Wohlbefinden an der Schule (emotionale Situation) scheint davon abzuhängen, ob und wie Lehrer und Klassenkameraden Rücksicht auf die besonderen Bedürfnisse der Jugendlichen mit Hörschädigungen nehmen. Eine Herausforderung stellen Lehr- und Lernmethoden, meist große Klassen und zu wenig Aufklärung über die Auswirkungen eines Hörverlustes dar.

Die Situation am Arbeitsplatz mit hörenden Kollegen akzeptieren einige Jugendliche als ihren „Alltag“. Sie wünschen sich, wie Normalhörende respektiert zu werden. Ihnen gelingt es, ihr Arbeitsumfeld so zu gestalten, dass sie sich darin wohl fühlen. Ein geringerer Teil berichtet über Probleme, die auf die Hörschädigung zurückgeführt werden.

Beim Wohlbefinden in Betrieb und Schule spielt die Persönlichkeit des Jugendlichen eine Rolle.

Ihre Leistung in der Berufsschule (leistungsmotivationale Situation) ordnen viele Jugendliche im Mittelfeld ein, einzelne sogar im vorderen Be- 
reich. Für einige ist dies mit einem Mehraufwand wie etwa erhöhter Konzentration im Unterricht und intensiver Nacharbeit verbunden. Ein Berufsschüler wechselte aus diesen Gründen an die Berufsschule eines BBW.

In der praktischen Ausbildung zeigt sich der überwiegende Teil mit der Leistung ebenfalls zufrieden. Ein Auszubildender gab sogar an, seine Arbeiten kompetenter und schneller auszuführen als seine Kollegen. Ein anderer brach jedoch die Ausbildung ab.

Bisher zeichnet sich ab, dass die Situation der Auszubildenden mit Hörschädigung interindividuell sehr heterogen ist und von verschiedenen äußeren und inneren Faktoren abhängt. Diese gilt es in weiteren Analyseschritten zu identifizieren.

Weitere Informationen sowie Literaturangaben können eingeholt werden bei markus.wild@edu. Imu.de

\section{Aufgabenbezogenheit von Kindern mit frühkindlichem Autismus im schulischen Kontext (Dissertationsprojekt)}

\section{Kristina Willmanns \\ Universität zu Köln}

\section{Problemaufriss}

Die Beschäftigung mit Lernaufgaben (,attend to task“, Holifield u.a. 2010, Teilaspekt von „academic engagement", Greenwood 2002) nimmt im schulischen Alltag eine zentrale Stellung ein. Neben der Aneignung fachspezifischen Wissens und Könnens wird grundsätzlich auch eine größtmögliche Selbsttätigkeit der Schüler angestrebt mit dem Ziel, „selbstständig-produktive Aneignungsaktivitäten“ (Müller 2010, 85) in Gang zu setzen. Die Fähigkeit, sich möglichst selbsttätig mit Lernaufgaben zu beschäftigen, wird verstärkt im Zusammenhang mit schulischen Lernund Leistungserfolgen diskutiert. „The ability to attend to tasks is a requisite skill for academic success in school." (Holifield u.a. 2010, 230)
Kinder mit Autismus-Spektrum-Störungen zeigen vielfach große Einschränkungen in ihren Fähigkeiten zum selbstständigen Handeln (Degner/Nußbeck 2011; Hume/Odom 2009). Dies äußert sich im schulischen Alltag z.B. in Schwierigkeiten, Lernaufgaben anzugehen, die Aufmerksamkeit auf wesentliche Inhalte zu richten oder zielorientiert den Handlungsprozess zu steuern. Selbstständigkeit in diesem Kontext meint, dass das Verhalten des Lernenden auf die Aufgabe bezogen ist (,ontask"), die Aufgabe folglich entsprechend der Anweisungen richtig bearbeitet wird und der Handlungsprozess ohne Hilfestellung (,absence of adult prompting") bewältigt wird (Degner/Nußbeck 2011; Hume/Odom 2007). Konkretisiert impliziert der Begriff Aufgabenbezogenheit (,ontask") jegliches Verhalten, das eine aktive oder passive Beschäftigung mit der Aufgabe beinhaltet (z.B. schreiben, zuhören, den Blick auf ein Arbeitsblatt richten,...) (Shapiro 2011). Die Fähigkeit, „on-task“ zu sein, das heißt sich konkret mit der Aufgabe zu beschäftigen, nimmt einen zentralen Stellenwert in der selbstständigen Auseinandersetzung mit schulischen Lernaufgaben ein.

In der Autismusforschung lässt sich in jüngster Zeit eine Auseinandersetzung mit dieser Thematik erkennen, deren Fokus primär auf intervenierende Maßnahmen gerichtet ist. Den Studien ist allgemein zu entnehmen, dass Kinder mit Autismus-Spektrum-Störungen bei der Aufgabenbearbeitung vor der Intervention tendenziell wenig on-task behavior zeigten und gleichzeitig hohen Unterstützungsbedarf aufwiesen (z.B. Degner/Nußbeck 2011). Schlussfolgernd wird auf die Wichtigkeit der Förderung und Unterstützung der Selbstständigkeit dieser Kinder verwiesen (Hume/Odom 2009).

Das Bearbeiten einer Lernaufgabe ist ein äußerst komplexer Handlungsprozess, der hohe Anforderungen an die Fähigkeiten zur Selbstständigkeit der Lernenden stellt. Ausgehend vom Handlungsphasenmodell (Achtziger/Gollwitzer 2010) wird der Prozess der Aufgabenbearbeitung bei verschiedenen Modellen der Selbstregulation in die drei Phasen der Vorbereitung (präaktional), der Handlung (aktional) und der Reflektion (postaktional) gegliedert (Landmann u.a. 2009). In allen Phasen hat die Aufgabenbezogenheit großen Einfluss auf die Selbstständigkeit im Handlungsprozess und den Handlungserfolg. Kinder mit einer Autis- 
mus-Spektrum-Störung können durchgängig Probleme im selbstständigen Handeln aufweisen, die mit den Beeinträchtigungen in den exekutiven Funktionen, einer schwachen zentralen Kohärenz und veränderten Aufmerksamkeitsleistungen in Verbindung gebracht werden (Degner 2011).

Es kann davon ausgegangen werden, dass die eingeschränkten Fähigkeiten zur Aufgabenbezogenheit einen wesentlichen Einfluss auf die Selbstständigkeit während der Beschäftigung mit Lernaufgaben ausüben. Der Mangel an Aufgabenbezogenheit und das hohe Bedürfnis nach Hilfestellung führen zu einer schlechten Ausgangssituation von Kindern mit Autismus-Spektrum-Störungen hinsichtlich schulischer Lern- und Leistungserfolge. Eine frühzeitige Förderung und Unterstützung dieser Kompetenz kann als eine bedeutsame präventive Maßnahme für die Entwicklung schulischer Basisfähigkeiten von Kindern mit Autismus-Spektrum-Störungen angesehen werden.

\section{Forschungsfragen und Zielsetzung}

Eine Auseinandersetzung mit der bisherigen wissenschaftlichen Erarbeitung dieser Thematik führte zu folgenden Schlussfolgerungen für die eigene Zielsetzung:

- In Interventionsstudien konnte gezeigt werden, dass Kinder mit Autismus-Spektrum-Störungen tendenziell ein geringes Ausmaß an Aufgabenbezogenheit aufweisen. Eine genauere Analyse dieser Beeinträchtigungen im Handlungsprozess unter einem qualitativen Blickwinkel wäre von großer Relevanz, um differenziertere Erkenntnisse über die Fähigkeiten, Bedürfnisse und Besonderheiten von Kindern mit Autismus-Spektrum-Störungen in der Aufgabenbezogenheit zu erlangen. Hieraus könnten wichtige Schlussfolgerungen für eine prozessbegleitende Diagnostik von Lernvoraussetzungen und Unterstützungsmaßnahmen gewonnen werden.

- Der Fokus lag bislang überwiegend auf experimentellen Studiendesigns. Folglich liegen kaum Erkenntnisse aus dem schulischen Kontext vor. Dies erscheint aufgrund der besonderen Bedingungen im Kontext Schule sowie des engen Zusammenhangs zwischen einer möglichst selbsttätigen Auseinandersetzung mit Lernaufgaben und schulischem Lernerfolg äußerst bedeutsam.

- „Engagement" von Kindern mit AutismusSpektrum-Störungen wird verstärkt auch im Zusammenhang mit verschiedenen Einflussfaktoren diskutiert (vgl. Ruble/Robson 2007). Besonders im schulischen Rahmen wird Handeln von einer Vielzahl von situativen, kontextuellen und sachlichen Faktoren begleitet, welche auf das Verhalten des Lernenden einwirken können. Eine Analyse dieser natürlichen Umgebungsfaktoren kann zu einem besseren Verständnis der Aufgabenbezogenheit von Kindern mit Autismus-Spektrum-Störungen im Kontext Schule beitragen.

Ausgehend von diesen Schlussfolgerungen können folgende Fragestellungen für die eigene Forschung formuliert werden, die im Speziellen die Aufgabenbezogenheit von Kindern mit frühkindlichem Autismus fokussiert.

1) Welche Fähigkeiten aufgabenbezogenen Handelns besitzen Kinder mit frühkindlichem Autismus?

Wie zeigt sich eine Aufgabenbezogenheit (on-task) bzw. eine Nicht-Aufgabenbezogenheit (off-task) im Verhalten der Kinder?

- Inwiefern wird Hilfe bzw. eine bestimmte Art von Hilfe benötigt? Wie äußert sich der Hilfebedarf im Verhalten der Kinder?

- Wie lässt sich das Verhalten im Laufe des gesamten Handlungsprozesses beschreiben und verstehen?

2) Wie kann das Verhalten unter Einbezug der verschiedenen Einflussfaktoren (kontextuellsituativ, personal-dispositional, sachlich-strukturell) gedeutet werden?

\section{Untersuchungsdesign}

Im Rahmen der qualitativ-explorativ ausgerichteten Untersuchung wird das Verhalten von $\sim 10$ Kindern mit frühkindlichem Autismus bei der Bewältigung von Lernaufgaben zu mehreren Zeitpunkten in ihrem Klassenkontext mittels Video aufgezeichnet. Geplant ist eine Datenerhebung bei Kindern im 2. bis 4 . Schulbesuchsjahr.

Entsprechend der Zielsetzung lässt sich die Auswahl und Konzipierung weiterer Erhebungsmethoden wie folgt darstellen: 
1) Erfassen der Aufgabenbezogenheit (on-task):

- qualitative Analyse der Videodaten

- evtl. adaptierte und erweiterte Version des BOSS - Behavioral Observations of Students in Schools (Shapiro 2011)

- Interview mit der/den Lehrperson(en)

2) Erfassen personal-dispositionaler Faktoren:

- Schülerfragebogen

- BRIEF: Verhaltensinventar zur Beurteilung exekutiver Funktionen, deutschsprachige Adaptation des Behavior Rating Inventory of Executive Function (BRIEF ${ }^{\circledR}$ ) (Drechslerl Steinhausen 2013)

3) Erfassen sachlich-struktureller Faktoren:

- Klassifikationssystem für Lernaufgaben

4) Erfassen kontextuell-situativer Faktoren:

Kontextanalysebogen

Die Stichprobenauswahl, Datenerhebung und -auswertung wird in einem zirkulären Vorgehen umgesetzt, wobei der Fokus bei der Stichprobengewinnung auf die Förderschulen mit dem Schwerpunkt körperliche und motorische Entwicklung gerichtet wird. Die Analyse und Triangulation der Daten erfolgt anhand ausgewählter qualitativer Auswertungsmethoden von visuellem und auditivem Datenmaterial.

Weitere Informationen und Literaturangaben können eingeholt werden bei: kristina.willmanns @uni-koeln.de

\section{Zur Bedeutung von Interessen- vertretung im Leben von Menschen mit Lernschwierigkeiten (Dissertationsprojekt)}

\section{Meike Nieß}

Universität Hamburg

\section{Hintergrund}

Während der letzten Jahre ist im Rahmen der Veränderungen innerhalb der deutschen Behindertenhilfe die politische Partizipation von behinderten Menschen immer mehr in den Fokus gerückt. Sie ist als zentraler Grundsatz und Ziel für alle Lebensbereiche in der UN-Behindertenrechtskonvention verankert (Hirschberg 2010). Im Zuge fachlicher Reformen und der Entwicklung von der institutionellen zur personalen Perspektive wird die Beteiligung von Nutzerlinnen zu einem der Kernpunkte der Veränderungen sozialer Dienstleistungen (Beck 2008). Dennoch ist politische Partizipation sowohl in der Fachdiskussion als auch als Handlungs- und Strukturprinzip professioneller Angebote bislang nicht umfassend verankert (Beck 2013).

Partizipation stellt neben Gleichheit und Freiheit einen der zentralen Begriffe in Theorie und Praxis der Politik dar (Hoecker 2006). Allgemein ausgedrückt handelt es sich bei politischer Partizipation um ,alle Tätigkeiten, die Bürger freiwillig mit dem Ziel unternehmen, Entscheidungen auf verschiedenen Ebenen des politischen Systems zu beeinflussen“ (Kaase 1987, 682). Es stehen also politische Entscheidungsfindungsprozesse im Mittelpunkt. Je nach demokratietheoretischem Hintergrund unterscheidet sich dabei das Verständnis der Reichweite des politischen Systems sowie der Formen und des Zwecks politischer Partizipation.

Der Dissertation liegt ein normatives Partizipationsverständnis zugrunde. Über die reine Durchsetzung von Interessen hinaus geht es auch „um Selbstverwirklichung im Prozeß [sic] des direkt-demokratischen Zusammenhandelns und um polit.-soziale [sic] Teilhabe in möglichst vielen Bereichen der Gesellschaft" (Schultze 2007, 398). Damit wird der Blick auf weite Teile des gesellschaftlichen Lebens ausgeweitet und Partizipation ist auch in lebensweltlichen $\mathrm{Zu}$ sammenhängen als inhärent politisch zu verstehen (Beck 2013). Partizipation stellt in einem solchen Verständnis nicht nur ein Mittel zur Entscheidungsfindung, sondern auch einen Wert an sich dar; sie dient im Zuge demokratischer Prozesse der Produktion individuellen wie auch öffentlichen Willens (Schmidt 1997). Hinter einem normativen Partizipationsbegriff steht ein Menschenbild, das von Subjekten ausgeht, welche die Befähigung zur politischen Beteiligung durch entsprechende Gestaltung von Bildungsprozessen erlangen können (ebd.). Zugleich stellt sich mit diesem - auch als material bezeichneten - Zugang die Frage nach der Umsetzung von Partizipation und den Bedingungen, 
unter welchen diese möglich wird (Hoecker 2006); auf der Ebene des Subjekts bedeutet dies insbesondere, Partizipationshandlungen in den Blick zu nehmen.

Die Frage der Umsetzung von Partizipation wird in diesem Forschungsprojekt exemplarisch hinsichtlich der Beteiligung von Menschen mit Lernschwierigkeiten an Formen der Interessenvertretung untersucht. Dabei wird der Begriff der Interessenvertretung als Überbegriff für Formen der Partizipation verwendet, die auch als Selbstvertretung, Mitwirkung, Mitbestimmung sowie Selbsthilfe bezeichnet werden, und schließt diese ein.

\section{Fragestellung und Zielsetzung}

Das Projekt setzt bei der Frage der Voraussetzungen und der Umsetzung politischer Partizipationshandlungen an. Im Fokus steht dabei die Form der Partizipation, welche für Nutzer/innen von Diensten der Behindertenhilfe oftmals einen zentralen Handlungsspielraum für Partizipation darstellt: die Beteiligung an kollektiven, formalisierten Formen der Interessenvertretung innerhalb und außerhalb von Einrichtungen der Behindertenhilfe. Zur Erfassung der Zusammenhänge hinsichtlich der Umsetzung von Partizipationsprozessen auf individueller Ebene werden die subjektive Bedeutung von Interessensvertretung sowie Bedingungen für die Beteiligung aus der Perspektive der Betroffenen untersucht. Dabei wird insbesondere der Zugang zu Interessenvertretungsgremien in den Mittelpunkt gerückt. Ziel ist es, ein Verständnis individueller Beteiligungshandlungen sowie der Bedingungen, die politische Partizipation für Menschen mit Lernschwierigkeiten überhaupt erst möglich machen, zu gewinnen und daraus Konsequenzen für professionelles pädagogisches Handeln im Hinblick auf die Unterstützung bedeutsamer Partizipationsprozesse abzuleiten.

Die folgenden Fragen sind dabei forschungsleitend:

Welche subjektive Bedeutung hat die Beteiligung an Interessensvertretungsmöglichkeiten im Leben von Menschen mit Lernschwierigkeiten?

- Welche Bedingungen für die Beteiligung werden als relevant erlebt?

\section{Forschungsdesign}

Für die empirische Bearbeitung der Fragestellung wurde eine qualitative Forschungsstrategie gewählt. Es wurden Leitfadeninterviews mit narrativen Anteilen geführt; nach einem offenen, autobiografischen Erzählstimulus zum Einstieg in das Gespräch folgten gezieltere, auf die Thematik Interessenvertretung ausgerichtete Nachfragen. Der Fokus lag dabei u.a. auf dem Eintrittsprozess in die Interessenvertretung, auf den im Rahmen der Tätigkeit durchgeführten Aktivitäten sowie auf der Bewertung des Engagements in den Gremien. Befragt wurden 25 Mitglieder acht verschiedener Interessenvertretungsgremien. Zu diesen zählen u.a. eine PeopleFirst-Gruppe, zwei Werkstatträte sowie mehrere ambulante und stationäre Wohnbeiräte. Das Sample ist sehr heterogen und beinhaltet sowohl Menschen, die einen sehr geringen Hilfeund Unterstützungsbedarf haben, als auch solche mit hohem Hilfebedarf und eingeschränkter lautsprachlicher Kompetenz. Die Befragten sind zwischen 24 und 62 Jahre alt, 14 von ihnen männlich, elf weiblich.

Aktuell findet die Auswertung der Daten statt. Als Auswertungsmethode wird ein kodierendes Verfahren genutzt, bei welchem über die Fallgrenzen hinweg eine thematische Analyse u.a. der Frage nach den Wegen in die Interessenvertretung sowie der Motive und Konsequenzen des Engagements möglich ist.

\section{Erste Ergebnisse}

Derzeit deutet sich an, dass verschiedene Bedeutungsdimensionen der Interessenvertretung herausgearbeitet werden können. Die Spannbreite der Motive reicht von eher persönlichen (Beispiel 1: „Das macht mir Lust. Das ganze macht mir Lust hier alles, ne, das ist, macht mir alles Freude hier.") bis hin zu eindeutig politischen Aspekten (Beispiel 2: „Und ich hab auch gemerkt, ähm, während, also ich, dass ich mich immer mehr für diese Arbeit interessier und dadurch auch ne politische Arbeit mir also entwickelt habe, also behindertenpolitisch arbeiten, darin auch gerne weiterarbeiten möchte."). 
Des Weiteren liegen die Einflussfaktoren, welche sich auf die Beteiligung an Möglichkeiten der Interessenvertretung aus der Perspektive der Befragten rekonstruieren lassen, auf verschiedenen Ebenen: Es können sowohl strukturelle, soziale als auch individuelle Faktoren identifiziert werden. Dabei erweist sich das soziale Umfeld als besonders einflussreich (Beispiel 3: „Also ich wurde angesprochen von meinem Gruppenleiter sag ich jetzt mal und der sagt: Du kannst doch so gut zuhören und gut dir alles merken und auch ziemlich alle, all, alles Mögliche verstehen. Und ja, dann hab ich gesagt: Ja, kann ich machen. Und dann dadurch ist es gekommen, dass ich auch Lust zu hatte, das zu machen ja und jetzt bin ich schon $n$ paar Jahre im Werkstattrat.").

Literaturangaben und weitere Informationen können eingeholt werden unter: meike.niess@ uni-hamburg.de

\section{Grammatikerwerb deutschsprachiger Kinder zwischen 4 und 9 Jahren (GED 4-9)}

\author{
Hans-Joachim Motsch, Lena-Maria Becker \\ Universität zu Köln
}

\section{Theoretischer Hintergrund}

Der Erwerb grammatischer Regelkompetenz ist bei der Entwicklung sprachlicher Fähigkeiten von besonderer Relevanz. Der Rückgriff auf morphologische und syntaktische Regeln dient als unverzichtbare Grundlage für die lautsprachliche und schriftsprachliche Produktion korrekter Sätze sowie das Verstehen von Sätzen und Texten. Dieser Zusammenhang wird erkennbar in den schulischen Leistungen: Kinder mit grammatischen Defiziten weisen massive Probleme beim Schriftspracherwerb auf und haben Schwierigkeiten beim Verständnis von Texten bzw. der Lehrersprache (Catts u.a. 2002; Motsch 2010).

Störungen und Blockaden beim Erwerb der grammatischen Regeln werden international als bedeutsamer Marker der spezifischen Sprachentwicklungsstörung angesehen (Leonard 1998).
Dies impliziert, dass Fehlentwicklungen im Bereich des Grammatikerwerbs von Kindern möglichst frühzeitig festgestellt werden müssen, um geeignete Förder- bzw. Therapiemaßnahmen ergreifen zu können und negative Auswirkungen auf den (schrift-)sprachlichen Entwicklungsbereich, auf eine erfolgreiche Sozialisation und auf die schulische und berufliche Laufbahn zu vermeiden.

Um dieser Forderung zu entsprechen, müssen Normdaten vorliegen, die die grammatische Entwicklung deutschsprachig aufgewachsener Kinder abbilden. Es muss auf gesichertes empirisches Wissen zurückgegriffen werden, das darlegt, in welchen Schritten und in welchem Alter der Erwerb des grammatischen Regelsystems erfolgt, damit Abweichungen und Störungen im Erwerb der grammatischen Fähigkeiten identifiziert werden können.

Obwohl das Phasenmodell von Clahsen (1986) auf diese Fragen bislang einige Antworten bezüglich der Erwerbsreihenfolge grammatischer Regeln geliefert hat, zeigt sich - belegt durch neuere empirische Befunde -, dass die auf Untersuchungsergebnissen von drei Kindern bis zum 4. Lebensjahr (Clahsen 1982) beruhende Einteilung der grammatischen Entwicklung in vielen Bereichen des grammatischen Regelerwerbs nicht haltbar ist. So lässt sich beispielsweise die Annahme, dass Kinder im Alter von vier Jahren auch komplexe grammatische Strukturen, wie die Kasusmorphologie, erworben haben und regelgeleitet anwenden können (Szagun 2007) durch die Untersuchungen von Popella (2005) und Maiworm (2008) an über 150 sprachunauffälligen, monolingual deutschsprachigen Erstklässlern nach der Einschulung widerlegen. Die Ergebnisse zeigen, dass viele sechs- bis siebenjährige Kinder Schwierigkeiten mit der Anwendung der Kasusregeln haben (Motsch 2009).

Wird der Fokus über die elementaren grammatischen Phänomene hinaus auf formal komplexere und kognitiv anspruchsvollere Strukturen wie z.B. das Passiv oder den Konjunktiv gerichtet, wird deutlich, dass die bestehenden Annahmen der Spracherwerbsforschung ohne empirischen Beleg existieren: Zwar herrscht Einigkeit über die Prozesse der Erweiterung und Ausdifferen- 
zierung des grammatischen Systems über das Kindesalter hinaus bis ins Jugendalter (Dannenbauer 2002), doch liegen den Beschreibungen dieser Erwerbsschritte keine methodisch abgesicherten Daten zugrunde.

Zusammenfassend gilt es festzuhalten: Die Erfüllung der Forderung nach einer frühzeitigen, von Normdaten abgeleiteten Einschätzung, ob der individuelle kindliche Grammatikerwerb normgerecht, verzögert oder gestört abläuft, scheitert aufgrund fehlender Daten. Es existiert kein ausreichend empirisch gesichertes Wissen über den Erwerb grammatischer Fähigkeiten deutschsprachiger Kinder (Motsch 2013).

\section{Ziel}

Das beschriebene beträchtliche Forschungsdesiderat im Bereich der grammatischen Entwicklung bildet den Ausgangspunkt für das Projekt GED 4-9. Im Rahmen einer großen multizentrischen Studie sollen repräsentative, empirisch zuverlässige Daten bezüglich des Grammatikerwerbs monolingual deutschsprachiger Kinder in der bislang kaum erforschten Lebensspanne zwischen dem vierten und neunten Lebensjahr erhoben werden.

\section{Methode}

Die Datenerhebung ist multizentrisch konzipiert und findet im Rahmen von drei koordinierten Teilprojekten in den Bundesländern Baden-Württemberg (GED 4-9 Baden-Württemberg; Leitung: AOR'in Dr. Margit Berg), Nordrhein-Westfalen (GED 4-9 Nordrhein-Westfalen; Leitung: Univ.-Prof. Dr. Hans-Joachim Motsch) und Niedersachsen (GED 4-9 Niedersachsen; Leitung: Univ.-Prof. Dr. Ulrike Lüdtke) statt.

Untersucht werden die grammatischen Fähigkeiten von ca. 1000 monolingual deutschsprachigen Kindern. Die Voruntersuchungen an einer Teilstichprobe $(n=110)$ wurden im September 2013 in Kitas und Schulen in Nordrhein-Westfalen durchgeführt. Die Hauptuntersuchungen erfolgen im Laufe des Jahres 2014 in den drei Bundesländern.
Um repräsentative Ergebnisse zu erlangen, werden bei der Stichprobenauswahl die Merkmale Alter, Geschlecht, Stadt/Land und Schulabschluss der Mutter berücksichtigt. Zur optimalen Durchmischung der Stichprobe erfolgt die Differenzierung nach Alter der Kinder in Viermonatsschritten. Die anschließende Berechnung der Normwerte erfolgt in Halbjahresschritten.

Im Zuge der Entwicklung des Erhebungsinstruments zur Überprüfung der grammatischen Fähigkeiten werden vorhandene Subtests des bestehenden Diagnoseinstruments ESGRAF-R (Evozierte Sprachdiagnose grammatischer Fähigkeiten; Motsch 2009) standardisiert und durch projektbezogen entwickelte Diagnostikmaterialien zur Überprüfung einzelner grammatischer Phänomene erweitert. Das daraus entstandene Erhebungsinstrument mit dem Rahmensetting "Zirkus“ erlaubt eine umfassende Beurteilung folgender grammatischer Fähigkeiten: Subjekt-Verb-Kongruenz, Verbzweitstellung im Hauptsatz, Kasusfähigkeiten (Genitiv, Dativ, Akkusativ), Genussicherheit, Pluralbildung, Verbendstellung im Nebensatz, Passivsätze, Konjunktivsätze.

Das Diagnostikverfahren wird im Rahmen der Voruntersuchung $(n=110)$ erprobt und hinsichtlich testtheoretischer Parameter wie z. B. Durchführungsgenauigkeit, Bestimmung des Schweregrads, Festlegung der Itemreihung usw. analysiert und anschließend gegebenenfalls für den Einsatz in der Hauptuntersuchung modifiziert. Die Pilotierung dient zudem der Validierung des Erhebungsinstruments durch ausgewählte Untertests bestehender Testverfahren (u.a. „Entwicklungstest Sprache 4-8 Jahre" [ETS 4-8, Angermaier 2007], „Sprachstandserhebungstest für Fünf- bis Zehnjährige" [SET 5-10, Petermann 2010], „Inventar diagnostischer Informationen bei Sprachentwicklungsauffälligkeiten" [IDIS, Schöler 1999]) sowie durch das Urteil der Erzieher/innen und der Lehrerlinnen über die sprachlichen Fähigkeiten der getesteten Kinder.

Die Interrater-Reliabilität wird über die Durchführung von ca. 20 - über die verschiedenen Alterskohorten gestreuten - Doppelauswertungen erfüllt. Der Nachweis der Test-Re-Test-Reliabilität 
erfolgt über die Errechnung der Stabilitätswerte nach einem Jahr. Diese Jahresnachtestungen werden im September 2014 an ca. 30 Kindern durchgeführt.

\section{Erwartungen}

Die erhobenen Daten schaffen erstmals Vergleichswerte zur Beurteilung der grammatischen Entwicklung im Vorschul- und frühen Schulalter von muttersprachlich deutschen Kindern.

Sie können die Fragen nach Erwerb und Anwendung der elementaren grammatischen Regelsysteme (z.B. Verbzweitstellung, Verbendstellung im Nebensatz) sowie der komplexeren Strukturen wie z.B. der Kasusmorphologie beantworten. Zudem liefern die Daten Erkenntnisse über formal komplexere und kognitiv anspruchsvollere grammatische Strukturen wie etwa die Verwendung von Passivkonstruktionen oder des Genitivs. Die bereitgestellten Normen erlauben dadurch erstmals den Vergleich des grammatischen Erwerbsstandes von Kindern mit Migrationshintergrund mit dem deutschsprachiger Kinder.

Sprachförderkräften, Erzieherlinnen, Lehrkräften und allen sprachpädagogisch und -therapeutisch tätigen Praktikerlinnen wird ein profundes Bewertungsmaß an die Seite gestellt, um die Grenze zwischen entwicklungsnormalen und gestörten grammatischen Fähigkeiten differenziert zu ziehen und gegebenenfalls von der normgerechten Entwicklung abgeleitete Förder-bzw. Interventionsmaßnahmen in die Wege zu leiten.

Das gewonnene Wissen erlangt besondere Bedeutung bei den Übergangssituationen und Schnittstellen unseres Bildungssystems - sowohl bei Sprachstandserhebungen bei Vierjährigen als auch bei Schuleingangsuntersuchungen und der damit verbundenen Entscheidung über Sprachförderbedarf.

Weitere Informationen sowie genaue Literaturangaben: j.motsch@uni-koeln.de 